The Impact of Large-Scale Surveys on Pulsating Star Research ASP Conference Series, Vol. 203, 2000

L. Szabados \& D. W. Kurtz, eds.

\title{
Ten Years of STEPHI Multisite Campaigns on $\delta$ Scuti Stars
}

E. Michel ${ }^{1}$, M. Chevreton ${ }^{2}$, J. A. Belmonte ${ }^{3}$, Li Z. P. ${ }^{4}$, M. Alvarez ${ }^{5}$, and the STEPHI Team

${ }^{1}$ Observatoire de Paris, DASGAL, UMR CNRS 8633, Meudon, France

${ }^{2}$ Observatoire de Paris, DAEC, UMR CNRS 8631, Meudon, France

${ }^{3}$ Instituto de Astrofisica de Canarias, La Laguna, Tenerife, Spain

${ }^{4}$ Beijing Astronomical Observatory, Beijing, China

${ }^{5}$ Universidad Nacional Autonoma de Mexico, Ensenada, Mexico

\begin{abstract}
STEPHI is a photometry network that aims to obtain data to reach a better understanding of the physics of stellar interiors by seismology of $\delta$ Scuti stars. For ten years now STEPHI has been harvesting multisite rapid photometry data of $\delta$ Scuti stars on the main sequence. With $12 \delta$ Scuti stars observed during three-week, three-site campaigns, STEPHI has produced a significant amount of the data available on $\delta$ Scuti stars for seismological study of this stage of evolution. The STEPHI data have already been basis of several theoretical inter-

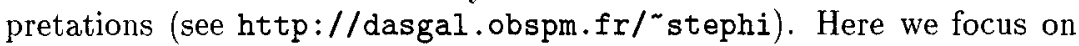
some aspects of our observational strategy and results.
\end{abstract}

\section{Characteristic Aspects of the Observational Strategy and Results}

-Selected targets: With the purpose to having as fruitful as possible returns in terms of theoretical interpretation, we hitherto focussed on main sequence objects (which have less dense theoretical oscillation spectra) with additional constraints for the modelling (e.g. $\delta$ Scuti stars in clusters or binary objects). -Three-site observations to suppress daily aliases ambiguity: Our threesite data are relatively free of this ambiguity; the first alias is currently lower than $40 \%$ of the central peak of the window function in amplitude (compared to more than $90 \%$ in single-site data).

-Noise level, the lower the better (but not at any price): With no lower limit being known for $\delta$ Scuti oscillations, we are seeking the lowest possible noise level. We currently reach a noise level of a few $\times 10^{-4}$ in the domain searched for frequencies, depending on the campaign, and depending on the sophistication of the reduction performed (see Table 1). This value of course depends on the intrinsic quality of the data merged together. An advantage of having more than three sites involved is that the best data at a given time can be selected, making it possible to obtain a higher quality time series without sacrificing dramatically the effective observational coverage. With three sites we are more sensitive to 
Table 1. STEPHI campaigns

\begin{tabular}{ccccccc}
\hline & Date & Object(s) & $\begin{array}{c}\# \\
\text { freq }\end{array}$ & $\begin{array}{c}\text { Noise level } \\
\langle P\rangle^{1 / 2}\end{array}$ & $\begin{array}{c}\text { Effective } \\
\text { duration }\end{array}$ & PI \\
\hline I & $06 / 87$ & 63 Her & 6 & & & J. A. Belmonte \\
II & $08 / 89$ & GX Peg & 5 & $1.5 \mathrm{E}-4$ & $219 \mathrm{~h}$ & E. Michel \\
III & $11 / 90$ & V650 Tau & 4 & $7.6 \mathrm{E}-4$ & $167 \mathrm{~h}$ & E. Michel \\
IV & $02 / 92$ & BU Cnc & 6 & $7.6 \mathrm{E}-4(3 . \mathrm{E}-4)$ & $228 \mathrm{~h}$ & J. A. Belmonte \\
& $/ /$ & BN Cnc & 5 & $7.6 \mathrm{E}-4(2 . \mathrm{E}-4)$ & $/ /$ & $/ /$ \\
V & $09 / 93$ & VW Ari & 7 & $6 . \mathrm{E}-4$ & $150 \mathrm{~h}$ & Liu Y. Y. \\
VI & $02 / 95$ & BQ Cnc & 9 & $4 . \mathrm{E}-4(1.7 \mathrm{E}-4)$ & $210 \mathrm{~h}$ & M. Alvarez \\
& $/ /$ & BW Cnc & 3 & $/ /$ & $/ /$ & $/ /$ \\
VII & $02 / 96$ & BS Cnc & 3 & $7.7 \mathrm{E}-4$ & $115 \mathrm{~h}$ & M. Hernandez \\
& $/ /$ & BT Cnc & 2 & $/ /$ & $/ /$ & $/ /$ \\
VIII & $11 / 97$ & V647 Tau & 5 & $1.6 \mathrm{E}-4$ & $228 \mathrm{~h}$ & E. Michel \\
IX & $11 / 98$ & V534 Tau & $\sim 11$ & $2.5 \mathrm{E}-4$ & $286 \mathrm{~h}$ & Li Z. P. \\
X & $12 / 99$ & in prep. & & & & M. Alvarez \\
\hline
\end{tabular}

* square root of the mean power level around $0.2 \mathrm{mHz}$. When different reduction procedures were used, the value given within brackets refers to the more sophisticated data reduction (using weighted FT or polynomial correction at low frequency), while the other value refers to the classical procedure.

meteorological conditions, and the result is a compromise between the noise level and the window function. However, as confirmed by the values presented in Table 1, the data regularly obtained by STEPHI are of prime quality and of the standard of the best data currently available. The noise can also be reduced by re-observing the same object several times. With the improvement going only as the square root of time, this can look costly. However, it is interesting to note that some examples exist in the literature where it has been possible to increase significantly the number of detected frequencies, some peaks being detected in one time series, but not in the others showing comparable noise levels.

-Two-colour photometry to help mode identification: We introduced two-colour measurements from one site in our latest campaigns.

\section{Conclusions}

During the past ten years, STEPHI has successfully completed its objectives: to obtain high quality data for a representative set of selected $\delta$ Scuti stars in order to allow theoretical interpretation in terms of stellar structure. These data have helped to shed new light on several aspects of the seismological interpretation of $\delta$ Scuti stars, both observational and theoretical. The set of $\delta$ Scuti stars for which such data are available is still very limited ( $\sim 20$ among 300 for reasonably well-covered multisite data over a couple of weeks). Since this kind of observation has a non-negligible cost in terms of telescope time and manpower, the international community surely has to co-ordinate its efforts. The physical return at stake is of prime interest for stellar physics. 